\title{
Moving XML to a Manufacturing Enterprise
}

\author{
Paul Lau, Jasper Wong, Edward Cheung \\ Industrial Centre, The Hong Kong Polytechnic University, Hong Kong \\ Email: icpaul@polyu.edu.hk
}

Keywords Information system reengineering, XML, SGML, Metadata, Data Source Object, Active Serve Page, Servlets, ODBC

\begin{abstract}
Information generation, representation and control in a manufacturing enterprise are often done through interactions of a variety of application software. Application software such as resource planning, electronic design automation, computer aided design and manufacturing, product data management, various database and supply-chain management applications are essential for an enterprise to be successful in this information age. For an enterprise to operate effectively, individual application must communicate and interact with each other, and user groups can share and exchange pools of information. Although client/server technology can break some of the barriers, with applications being shared among common databases and open system platforms, it does not imply that the applications are capable of inter-operating data and document can be efficiently managed and distributed over the manufacturing enterprise at large. XML provides a tool to integrate data from different sources and eliminate client-extensions to achieve universal access. In this paper, we will incorporate XML in a manufacturing enterprise to provide a cross-organizational support for a board range of users, across hardware and software boundaries conveniently and cost effectively.
\end{abstract}

\section{INTRODUCTION}

Information management is a key factor for the success of a manufacturing enterprise. The enterprise resources have unleashed the current technological revolution that has fuelled this Information Age. Internet technologies applied to build corporate intranets and also extranets between customers, suppliers, and business partners have opened up heterogeneous access leading to manufacturing intelligence. An effective manufacturing enterprise needs a modern and flexible data delivery system so as to access both structured and unstructured data in the enterprise 
databases. Most of these databases exist as unstructured data that include textual documents, reports, graphics, audio and video resources. Developing after the successful application of Hypertext Markup Language (HTML), Extensible Markup Language (XML) is an Internet technology that can be applied to document metadata used between different systems. It helps in organising and locating data [1]. XML is a data format for information interchange on the Web [2]. In addition, it acts as a bridge between structured and unstructured data. Metadata can be published in a Document Type Definition (DTD) file, which defines the structure of an XML file. XML can be used for describing data of virtually any types. It is a language for creating mark up. It is more flexible and powerful than HTML. It is supported by most of the productivity tools and office suites [3]. It offers an effective way to provide communication between different databases systems and platforms.

The XML technology offers two structuring methodologies, Heavyweight structuring and Light-weight structuring, to define information structure of documents [4]. Heavy-weight structuring applies in a way similar to designing data structure for programming. It consists of complete sets of syntax rules, constraints, attributes, tags and semantics. The structure of the documents plays an important part in the delivery of document information. Light-weight structuring applies when information is best expressed in natural texts. The markup of a particular key-sentence in a content page helps readers to identify vital information and it is the key to success for the delivery of information in the enterprise. Instead of defining a complete document model, markup identify elements, add sentinel marks or express semantic links with the documents.

\section{XML DATA MAPPING}

XML-data is closely related to the MCF specification [5], and can be applied for structured data and metadata exchange on the Internet. It describes an XML vocabulary for defining and documenting object classes. It can be used for classes that are strictly syntactic or indicate concepts and relations among concepts as in relational databases [6].

An XML document is primary concerned with data and the document is a data source. XML is a universal data format for structured document. It is text-based and platform independent. Different applications and HTML pages can access and manipulate a relational database once it is converted into an XML data source. One can convert a Microsoft Access table into a XML based data by accessing the data source through scripts or Data Source Objects (DSO). Another method is to transform the table, using the 
Active Server Pages (ASP) technology, into an XML data source. In this way, Web or Intranet users can access this information more quickly. By using this method, XML data source can be generated and updated dynamically. This data conversion can be achieved through some simple mapping methodologies between XML and objects or between XML and tables [7]. Figure 1 shows that there is a one-to-one relationship between the object model and the XML model.

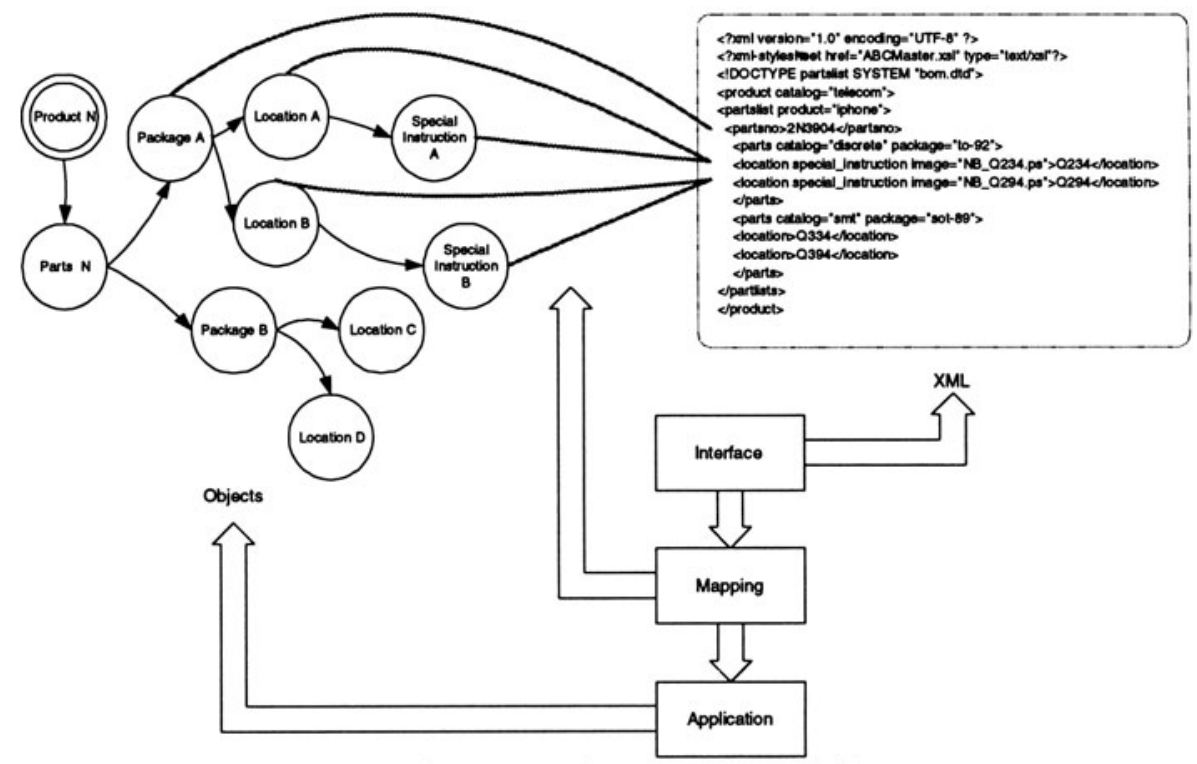

Figure 1 Mapping between XML and objects

As the object model and the XML model are similar, the Mapping between the XML and objects are simple. If you parse XML, you can obtain a tree of common data structure for the Objects. Figure 2 shows a Mapping between XML and tables. Since the two models are different, and it is not a one-to-one relationship, the Mapping between XML and the relational tables is more complicated.

\section{THREE TIER MODEL FOR XML}

\subsection{XML and Databases interface}

Figure 3 shows a Java based Three-tier Model which consists of a Web Browser Client as tier1, a Web Server as tier 2 and a Backend Server as tier 3. The most common Backend Servers are Relational database management systems (RDBMs). A Backend Server is the data source for enterprise legacy applications. XML web applications exchange information between 
organizations through this database access architecture. The RDBMs are very efficient in dealing with large amount of data. It is difficult to exchange data between RDBMs. XML offers a flexible and standard way for data exchange. The middle tier is a web server to provide connectivity for web clients and business logic support in the manipulation of data from the databases. In Java enabled system, a Servlets enhanced Web Server can be used. Servlets are effective in providing secured Web-based application which normally requires interaction between databases and clients. It can also generate dynamic and generate custom HTML documents for display while maintaining unique session information for each client. Using Servlets, input/output streams and Java Database Connectivity (JDBC), robust multi-tier client-server applications that access databases can be built.

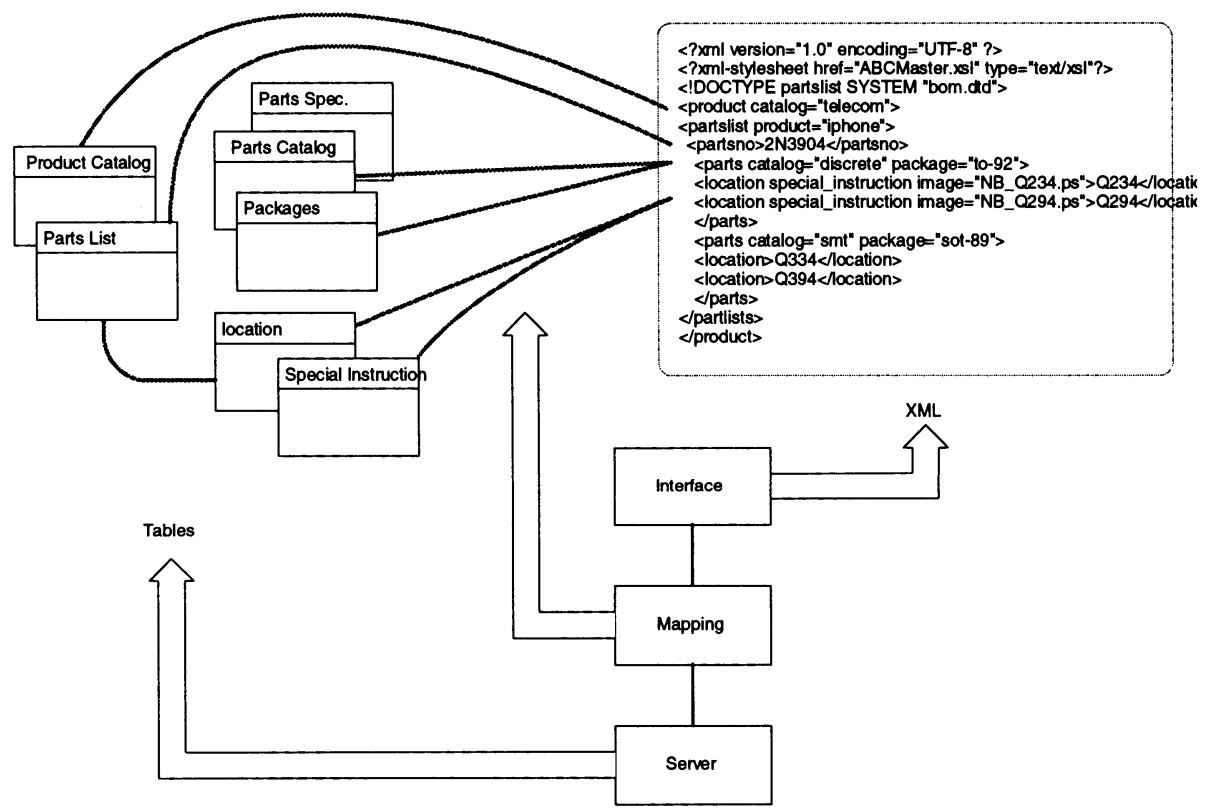

Figure 2 Mapping between XML and tables

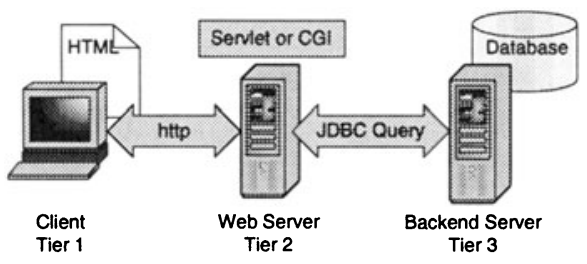

Figure $3 X M L$ and Database Interface 


\subsection{Application Model}

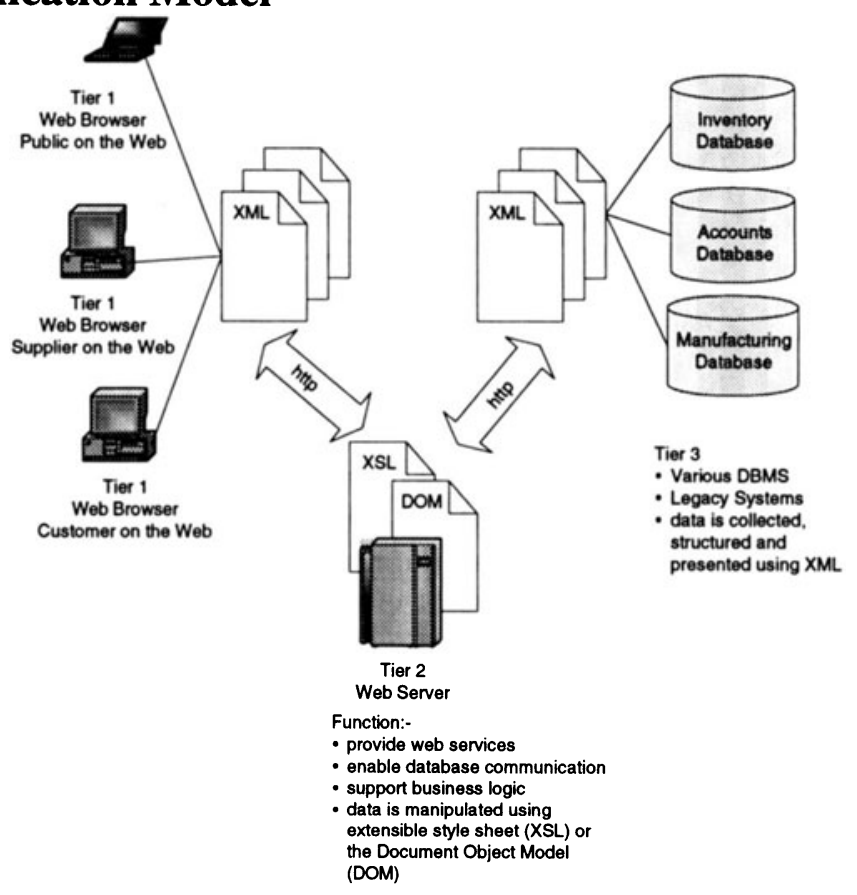

Figure 4 XML Three-tier Application Model

In most organizations, management database is normally stored in a proprietary file system. It doesn't make sense to convert all the existing data to XML format because individual application has its uniqueness and schema. For security reasons, most of the manufacturing enterprises do not want to expose unnecessary information on the Internet. Hence, it is necessary to isolate the database from the Internet clients. Figure 4 shows a typical XML three-tier application model. Tier 3 consists of a set of legacy systems and databases. Structured data are entered into or retrieved from these systems by the enterprise users. The existing data in the databases stays in its current format. In tier 2, structured information is compiled or converted to XML raw data. The XML data will be exchanged over HTTP to the Web client in the tier 1 . Together with the XML data, a style sheet can be created for the Web client to download and display information in the original format from the content provider. The Web client can then perform editing, rendering, calculations, graphical interpretation, statistical processing and other manipulation on the XML data independently. In this way, web client can take advantages of their hardware rather than depending on the processing power as distributed by the server. 


\section{INFORMATION MANAGEMENT SYSTEM FOR A MANUFACTURING ENTERPRISE}

The use of information system has been increasingly playing a critical role towards the demand on productivity and throughput in a manufacturing enterprise. Figure 5 shows an information process within a manufacturing enterprise.

The enterprise, in general, consists of sales and marketing, design, process planning, production, material control and purchasing functional units. Manufacturing information and documents are managed in traditional databases, drawing files, office application, bitmap images, vector images together with different databases, which cater for a particular manufacturing system or process. Integration for these manufacturing information and documents are necessary. The primary drive for this integration is the demand in efficiency, collaboration and quality in an organisation. These demands cannot be met by partial automation in individual process. Collaboration tool is essential because it can integrate all documentation and databases. With a good user interface, the collaboration tool can provide a convenient environment to improve co-ordination between business functions and improve decision-making. Many problems and issues relating to the design, development, integration, evolution and maintenance of Information System in a large scale and complex plants have become apparent and are not adequately addressed by the traditional process. A sophisticate information system makes a manufacturing enterprise ideal for manufacture-to-order, assemble-to-order, configure-to-order and engineerto-order. This flexibility is essential for a manufacturing enterprise to success in the Internet business.

A collaborative information system enables the management team to track and report on all activities, integrates materials and costs which associated with individual products with entire manufacturing functions or business function. Through this information system, the enterprise management makes comprehensive order processing and inventory information available for immediate analysis. The management can analyse customers' buying patterns, track the performance of items and identify market opportunities that can shape future marketing activities. It supports international quotes and sales orders and allows the management of sales contracts anywhere anytime. Purchasing is fully integrated with all manufacturing and financial function so as allows the enterprise to reduce its inventory for a more profitable operation. This system can also provide 
formatted information for the suppliers. This will enable collaboration not only limited within an enterprise itself, it virtually extends its business boundary. The enterprise can set up strategically supply chain or partners without worrying the incompatibility of systems. Manufacturing enterprise is normally supported by different systems introduced during various stages in its development. These may include resource planning, electronic design automation (EDA), computer aided design(CAD) and manufacturing (CAM), product data management, decision support and data warehouse and supply-chain management. By integrating with different objects or DTD, the enterprise information system provides the capability to integrate information reside in different RDBMS such as engineering drawings in CAD/CAM systems with resources management systems such as MRP.

\section{AN IMPLEMENTATION EXAMPLE}

$\mathrm{ABI}$ is a manufacturing enterprise. It has more than twenty sales offices and a factory over the world. Manufacturing information such as cost, shipment, product specifications, production rate, stocks and parts need to exchange between the factory and sales offices frequently. There are over a hundred products for the $\mathrm{ABI}$ enterprise. It is very common to produce over twenty different products in a day. The factory implements a Just-in-time inventory system and multi-vendors policy. Hence, a tight interaction between the factory, vendors and the sales offices is very important. ABI enterprise has a long history. Its information systems and databases have evolved over many years. Consequently, it suffers from loose coupling in its information system with isolated or proprietary processes that operates in different platforms and legacy DBMS. Management information exists as word processor files, spreadsheets, legacy relational databases, engineering drawing, sales and suppliers catalogues that have different formats and metadata. Under the current environment, information is neither coalesced nor intelligent.

Figure 6 shows a proposed Information Architecture for the ABI enterprise using XML technology. It is based on the Three-tier Model that we previously discussed. It consists of three parties: vendors, sales offices and the factory. The clients are the end users from the vendors, sales offices and the factory. One the client sides, data is formatted by the Data Source Object (DSO) scripting. Information is sent to the Internet Explorer 5 browser from the factory's Internet Information Server. 


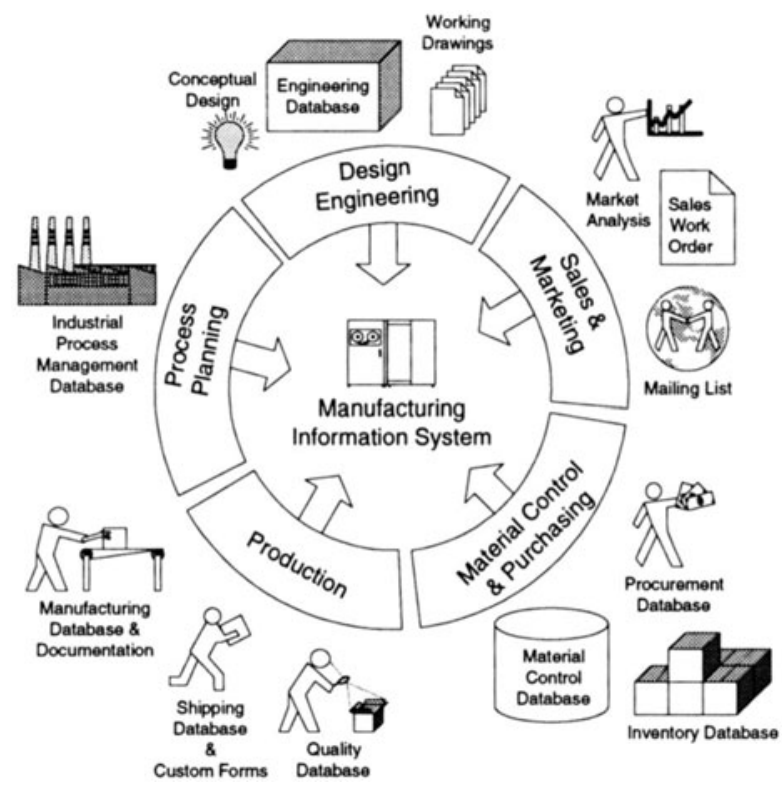

Figure 5 Information Process in a Manufacturing Enterprise

In addition to a Web server, the middle tier comprises an INSIGHT XML database and the INSIGHT software system [8], which automatically combines document and graphic-based information from databases and file systems into intelligent Web applications. Whenever a customer places an order to a sales office, a salesman can issue query to the Business Server from the browser. Figure 7 shows part of the XML sample codes implement in this application.

\section{CONCLUSION}

In this paper we have discussed some key features of XML as a tool to integrate data from different sources as well as to achieve a universal data and document access. It shifts the process of data visualisation from a server to the client and allows various data manipulations at the client side. It is a convenient way to publish document and exchange data either internally or externally in a manufacturing enterprise context including Internet access for its strategic partners. The XML technology offers Heavyweight and Lightweight structuring methodologies through data mapping from data sources. This facilitates the creation of XML documents from various data formats. 


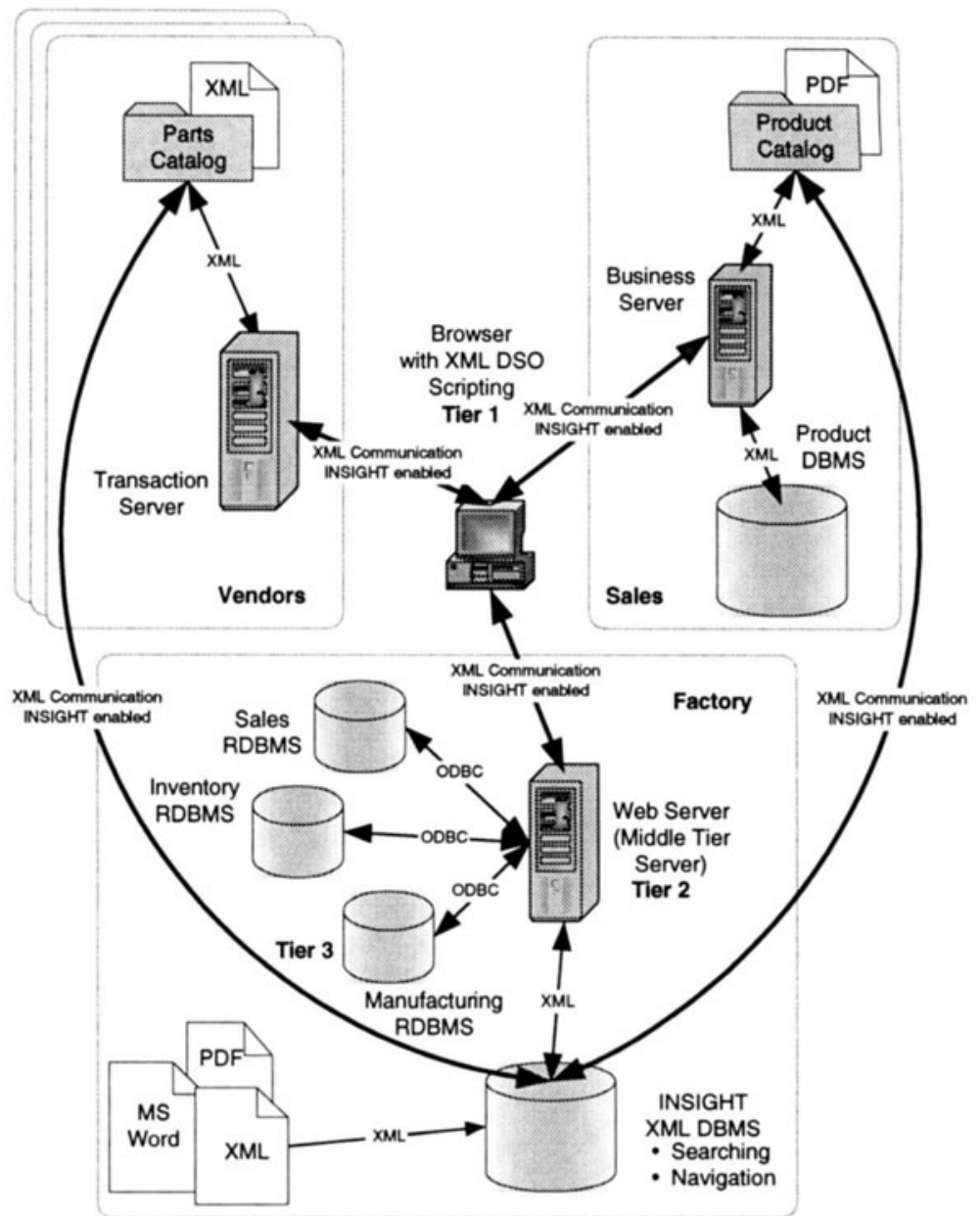

Figure 6 Manufacturing Enterprise Information Architecture

A Three-tier model was introduced in this paper. The model can be applied in any manufacturing enterprises. As information flow and system is typical complicated and important in such environment, it is essential to move the XML technology to the enterprise safely and seamlessly with minimal disturbance. In our application, we have illustrated that moving the XML technology to the manufacturing enterprise can seamlessly improved its information system and hence the productivity and the profitability of the enterprise. An example has been discussed to demonstrate the implementation of a XML enabled information system in a manufacturing enterprise. By incorporating XML in a manufacturing enterprise, cost effective information flow can be achieved across various hardware and 
software platforms. A manufacturing enterprise can run ahead of its competitors in this information age.

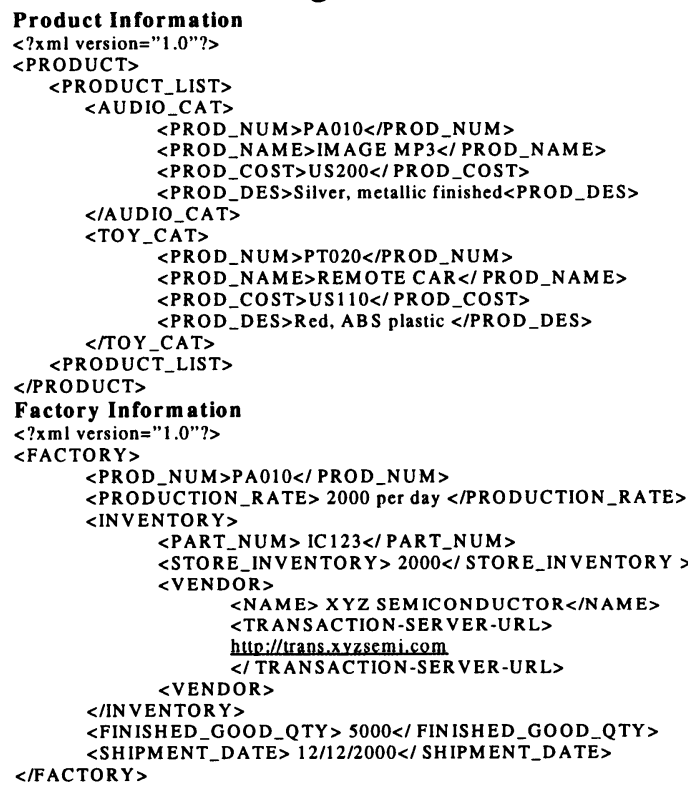

Figure 7 XML Sample Code

\section{REFERENCES}

[1] Scott Mace, Udo Flohr, Rick Dobson and Tony Graham(1988). Weaving a better Web: Byte Magazine, March 1998, pp. 58-68

[2] Extensible Markup Language (XML) 1.0W3C Recommendation, February 1998. http://www.w3.org/TR/1998/REC-xml-19980210

[3] Widergren S., deVos A., X J. (1999). XML for Data Exchange: Power Engineering Society Summer Meeting, IEEE Vol. 2, 840-842

[4] Bapst F., Vanoirbeek C. (1999). XML documents production for an electronic platform of requests for proposal: Reliable Distributed Systems, Proceedings of the $18^{\text {th }}$ IEEE Symposium, 330-308

[5] Meta Content Framework using XML (MCF), Specification (1997) http://www.textuality.com/sgml-erb/w3c-mcf.html

[6] Dianne Kennedy (1999), "Introducing XML-Data", XMLperts Articles library http://www.xmlxperts.com/xmldata.htm

[7] Barry D. (1999). XML Data Servers: An Infrastructure for Effectively Using XML in Electronic Commerce, Barry \& Associates, Inc. http://xmml-data-servers.com

[8] INSIGHT: "White paper: Enigma's e-Publishing Product Suite" http://www.enigma.com 